\title{
OTIONOMICS
}

Revista de economía, empresa y sociedad

Dossier «Realidades y desafíos de la Unión Europea»

CAMBIOS RELEVANTES

\section{La economía política de las industrias de red en la Unión Furopea}

\section{Francesc Trillas}

Profesor agregado del Departamento de Economía Aplicada de la Universidad Autónoma de Barcelona

RESUMEN Las industrias de red han experimentado cambios muy importantes en las últimas tres décadas en todo el mundo, y previsiblemente seguirán experimentándolos, impulsadas por cambios tecnológicos y económicos y también por las luchas de grupos de interés enmarcadas por parámetros institucionales e ideológicos. En la Unión Europea los cambios han ido acompañados por una creciente implicación del nivel comunitario, sin llegar a la creación de redes europeas. Paradójicamente, puede ser beneficioso para la resolución de los dilemas que se presentan en el sector eléctrico que no esté demasiado desarrollado un demos europeo, aspecto que se presenta problemático desde otros puntos de vista. Un demos europeo consolidado implicaría la existencia de una arena política europea arraigada, con una opinión pública europea, unos partidos políticos europeos, unos lobbies operando a nivel europeo... La razón por la cual esta falta de desarrollo del demos europeo puede ser favorable en sectores como el eléctrico o el de las telecomunicaciones es que esto permitiría despolitizar las intervenciones públicas en este tipo de sectores. A pesar de que la Unión Europea ha desempeñado ya un papel importante aumentando la competencia en estos sectores, puede tener un papel mucho más importante en el futuro avanzando hacia un auténtico mercado integrado donde existan redes realmente de alcance europeo.

PALABRAS ClAVE telecomunicaciones; electricidad; Unión Europea; economía política

\section{The political economy of network industries in the European Union}

ABSTRACT In the last three decades, network industries have experienced major changes the
world over. It can be presumed that they will continue to do so, driven by technological and eco-
nomic changes and also by the campaigns of interest groups framed by institutional and ideological
parameters. In the European Union these changes have seen a growing degree of involvement at
the community level, without reaching the point of creating European networks. Paradoxically, this
could be beneficial for the resolution of certain dilemmas that arise in an electricity sector where a
European demos is not well established, a factor that is problematic in other aspects. A consolidated
European demos would require a consolidated European political arena, European public with Eu-
ropean political parties and lobbyists working at a European level, and so on. The reason this lack of 
development of a European demos may be favourable to sectors such as electricity or communications is that it can stop public interventions in these sectors from being made into political issues. Although the European Union has already played an important role in increasing competition in these sectors, it may play a much more important role in a future that advances towards a market that is truly integrated, in which networks of a real European scope exist.

KEYWORDS telecommunications; electricity; European Union; political economy

\section{Introducción}

El logro del objetivo de combinar prosperidad e integración que tiene planteado Europa depende, entre otros factores, de un buen funcionamiento de aquellos sectores relacionados con las infraestructuras y las industrias de red. En este artículo se analizan en consecuencia algunos de los retos a los cuales se enfrentan estas industrias, en concreto las telecomunicaciones y la electricidad, en la Unión Europea.

Estas industrias han experimentado cambios muy importantes en las últimas tres décadas en todo el mundo, y previsiblemente seguirán experimentándolos, impulsadas por cambios tecnológicos y económicos y también por las luchas de grupos de interés enmarcadas por parámetros institucionales e ideológicos. Tradicionalmente, tanto las telecomunicaciones como la electricidad eran suministradas por empresas verticalmente integradas (por ejemplo, una misma empresa generaba, transportaba y distribuía electricidad) de tipo monopolístico en un determinado territorio (correspondiente a todo o parte de un estado-nación, pero no en más de uno), en general propiedad del estado (pero no únicamente). Dado que eran considerados monopolios naturales, es decir, sectores donde por razón de costes era conveniente que operara una sola empresa, las empresas operadoras eran objeto de una intensa regulación. Los cambios ocurridos en las últimas décadas se han centrado en una intensa reestructuración, que ha implicado elementos de separación vertical, introducción de competencia y privatización en diferentes grados.

En el caso europeo, estas reformas han coincidido en el tiempo con la expansión de la Unión Europea hacia los países del este y la profundización de la Unión, con la creación de la moneda única y la intensificación en algunos aspectos, pero no en todos, del proceso de integración y creación de un mercado único. Uno de los aspectos donde el órgano ejecutor de las políticas comunitarias, la Comisión Europea, se ha mostrado más activo ha sido en formas microeconómicas de intervención pública, como la política de defensa de la competencia y los paquetes regulatorios en telecomunicaciones y electricidad. La dimensión europea en las industrias de red es importante: todos los niveles de la cadena vertical de gobierno están implicados en las políticas públicas que les afectan.

En el segundo apartado del artículo se presentan algunas características fundamentales de las industrias de red y se explican las recetas habituales de políticas públicas que de ellas se derivan. En el tercero se presentan algunas cuestiones que pueden ser útiles en cuanto a aspectos federales de la regulación. Y, a continuación, en el apartado cuatro se analizan los cambios experimentados en la Unión Europea en cuanto a telecomunicaciones y electricidad. Finalmente, el quinto apartado presenta unas breves conclusiones.

\section{Recetas convencionales sobre las industrias de red}

Las industrias de red, como las telecomunicaciones y la electricidad, se caracterizan por una estructura vertical compleja, donde coexisten segmentos con un diferente potencial de introducción de competencia. Son sectores en los que el carácter modular es fundamental: para poder producir el servicio, tienen que intervenir diferentes empresas, gobiernos e instituciones, y no hay una única manera de combinar estas diferentes entidades. 
En un extremo, hay segmentos que son monopolios naturales, los más característicos de los cuales son los de transporte y distribución de la electricidad. En el otro extremo, hay segmentos que son competitivos, pero con un tipo de competencia imperfecta, dado que habitualmente hay un número limitado de operadores. Esto hace necesario que estos segmentos sean objeto de atención por parte de la políica de defensa de la competencia.

La relación entre segmentos monopolísticos y segmentos potencialmente competitivos es compleja y presenta retos competitivos e institucionales relevantes, especialmente en cuanto a posibilitar el acceso de las empresas potencialmente entrantes a los segmentos monopolísticos de la empresa tradicionalmente establecida en el sector.

Dadas las características de las industrias de red, el mercado como mecanismo único de asignación de recursos resulta muy imperfecto, debido a la presencia de elementos monopolísticos, de externalidades positivas y negativas y de asimetrías de información, razón por la cual las políticas públicas desempeñan un papel muy importante en este tipo de sectores. Dado que las imperfecciones del mercado son más de una y afectan a diferentes segmentos de la cadena de valor, las políticas públicas que se deben desarrollar serán diversas y la relación entre ellas compleja: estas políticas incluyen la ya mencionada política de defensa de la competencia, la regulación ex-ante de algunos precios y condiciones del servicio y las ayudas públicas para desarrollar políticas de servicio universal.

La necesidad de arbitrar conflictos entre diferentes productores y diferentes consumidores da lugar a problemas distributivos, que se añaden a los problemas de eficiencia que hay implícitos en las nociones tradicionales de imperfecciones de los mercados. A pesar de que sería conveniente poder separar nítidamente la solución de los problemas de eficiencia y los problemas distributivos, utilizando instrumentos diferentes de políticas públicas, esto no siempre es posible porque no siempre todos los instrumentos teóricamente posibles están a disposición de los responsables públicos con el mismo grado de facilidad.

Pero la actuación pública también es imperfecta. En primer lugar, se dan problemas derivados de la inevitable politización de estos sectores, tratándose de realidades donde toda la población votante es consumidora y donde las empresas productoras tienen mucho en juego, se produce el riesgo de captura del regulador. En segundo lugar, las autoridades reguladoras suelen tener menos información sobre costes y demanda que las empresas operadoras.

Una característica importante de las industrias de red es el carácter específico, hundido, de las inversiones que son necesarias. Una inversión es específica, por oposición a genérica, cuando, una vez realizada físicamente la inversión (por ejemplo, una vez construida una central nuclear), los activos no se pueden ocupar en mercados o actividades alternativas, sino que quedan inmovilizados en su situación y ocupación actual durante décadas. Esto deja a los inversores, una vez realizada la inversión, en una posición vulnerable, dado que el activo puede operar sin que necesariamente se remuneren todos los costes fijos. Por su parte, los reguladores pueden verse presionados por opiniones públicas con importantes problemas sociales, que pueden desear disfrutar de los servicios que puede proporcionar el activo hundido sin necesariamente pagar los precios que remunerarían la inversión. Los inversores no pueden amenazar con llevarse la inversión a otro lugar. Esto da lugar a un problema de compromiso regulatorio: si los inversores no están seguros de que la regulación les compensará por las inversiones, serán muy reticentes a realizarlas en primer lugar, ya que anticiparán la posible expropiación en la práctica de los activos por la vía de unos precios regulados demasiado bajos, u otros tipos de políticas públicas que hagan no rentable la inversión realizada. A lo largo de la historia, las sociedades han desarrollado diferentes mecanismos para resolver este problema de compromiso, y lo más habitual históricamente ha sido la propiedad pública de las empresas en las industrias de red. La fuerte presencia de activos específicos es una característica que se mantiene a lo largo de la historia de las industrias de red. Por lo demás, la tabla 1 resume las diferencias entre las características tradicionales y más recientes de estos sectores.

Tabla 1

\begin{tabular}{|l|l|l|l|}
\hline & \multicolumn{1}{|c|}{ Estructura vertical } & \multicolumn{1}{c|}{ Competencia } & \multicolumn{1}{c|}{ Propiedad } \\
\hline $\begin{array}{l}\text { Características } \\
\text { tradicionales }\end{array}$ & Integración & No & Más pública que privada \\
\hline Características actuales & $\begin{array}{l}\text { Separación (no total) de algu- } \\
\text { nos segmentos }\end{array}$ & Sí, en algunos segmentos & Más privada que pública \\
\hline
\end{tabular}

Fuente: Elaboración propia. 
Majó (2015) da un buen ejemplo de lo que son las recetas estándar para conseguir eficiencia combinada con equidad en el sector: hacer pagar a los usuarios por los costes variables de producción y cubrir los costes fijos mediante transferencias públicas, idealmente financiadas con impuestos no distorsionantes o como mínimo progresivos. En coherencia con esto, la propiedad de aquellos segmentos donde se centran los elementos de costes fijos (los segmentos de infraestructuras, que son monopolio natural) podría ser fundamentalmente pública. En la práctica, las economías de alcance entre segmentos monopolísticos y competitivos y la ausencia de instrumentos impositivos no distorsionantes dificultan la aplicación de estas recetas convencionales, por lo cual encontramos en diferentes países distintas maneras de aproximarse (o no) a este ideal, como se explica en Briglauer et al. (2014).

En Europa, el mecanismo elegido para intentar superar el problema del compromiso regulatorio ha sido el de las agencias reguladoras independientes del gobierno, si bien estas han operado con un nivel de independencia variable entre países y a lo largo del tiempo. Por ello, a las recetas canónicas se ha añadido en los últimos años la de crear agencias reguladoras independientes del gobierno para asegurar que la regulación supera el problema del compromiso regulatorio e incentiva niveles adecuados de inversión más allá de la coyuntura política.

\section{Federalismo regulatorio}

En Trillas (2008) se analiza con detalle cuáles son las implicaciones de tipo positivo y normativo en cuanto a la organización de la regulación de las industrias de red a lo largo de los diferentes tipos de gobierno, desde el nivel local hasta el global. Esta ha sido una cuestión crucial en grandes agregados democráticos de carácter federal, como Estados Unidos, y lo está siendo en la Unión Europea.

Wallace Oates desarrolló el principio de subsidiariedad, según el cual las políticas públicas se tienen que desarrollar en el nivel más pequeño que agota las externalidades relevantes. Las consideraciones de Oates apuntan a ubicar cada responsabilidad en el nivel de gobierno geográficamente óptimo. Considerar cuál es el alcance geográfico del mercado o de la imperfección del mercado (por ejemplo, de un bien público) es un ingrediente importante a la hora de decidir en qué nivel de gobierno ubicar una responsabilidad de regulación. La heterogeneidad de las preferencias entre territorios también es un aspecto importante y combinada con el tamaño del mercado o del problema que hay que considerar puede dar lugar a un tamaño óptimo de la jurisdicción regulatoria, que no necesariamente coincidirá con las fronteras de las jurisdicciones tradicionales (como sucede en Estados Unidos con los mercados mayoristas de electricidad o con los distritos de distribución de agua).

Consideraciones políticas pueden alterar la decisión de cuál es el nivel jurisdiccional óptimo de la regulación. El historiador económico Werner Troesken, investigando la industria del gas en Illinois a finales del siglo XIX, llegó a la conclusión de que en Estados Unidos se había producido en aquella época un traspaso de capacidad regulatoria de los municipios hacia los estados. La razón era que la politización de la regulación de las public utilities a nivel local era tal, que se hacía imposible llegar a soluciones estables que garantizaran la inversión y la prestación del servicio con buenos niveles de calidad. En cambio, las agencias reguladoras de los estados con posterioridad fueron capaces durante muchas décadas de lograr unos niveles de estabilidad regulatoria elevados, lejos de la politización local que se había producido anteriormente. Troesken advierte sin embargo de que en la segunda mitad del siglo xx esta politización, y la aparición de elementos de captura de la regulación y de corrupción, se reprodujeron, por lo que aparecieron fuertes movimientos desreguladores e incluso de regreso de la regulación al nivel local.

Los problemas que interactúan en sectores como las telecomunicaciones y la electricidad afectan desde la escala local hasta la escala global, y requieren la intervención coordinada de los diferentes niveles de gobierno, en un grado e intensidad que depende del sector, la herencia institucional, el espacio y el tiempo. 


\section{La integración europea y las industrias de red}

Los cambios que se han producido en los últimos treinta años en las industrias de red de todo el mundo han coincidido en la Unión Europea con el proceso de expansión e integración de la Unión. Se dan paralelismos interesantes con la política monetaria y financiera, donde la necesidad de reaccionar ante la crisis de 2008 también ha coincidido con los primeros pasos del funcionamiento de una moneda única.

Como se explica con más detalle en Trillas (2010), se ha producido en las últimas décadas una progresiva toma de responsabilidad de las autoridades europeas (Parlamento y Comisión) en cuanto a la regulación de las telecomunicaciones y la electricidad, con hasta tres paquetes regulatorios en cada caso que han ido ampliando el alcance y la ambición de las políticas europeas. En ambos casos, el acento ha recaído progresivamente en una creciente liberalización de los dos sectores (en paralelo a una fuerte política comunitaria de defensa de la competencia), ampliando el alcance y las posibilidades de la competencia entre operadores. El carácter prescriptivo de las prácticas a desarrollar ha sido mucho más extenso en el caso de las telecomunicaciones, donde las directrices comunitarias son mucho más detalladas sobre qué tienen que hacer las autoridades de los Estados miembros en cada mercado y donde se produce un proceso de codecisión cuando se observa que en un determinado mercado de producto se produce una excesiva posición dominante. A pesar de que en electricidad el tercer y último paquete aprobado ya obliga a separar empresas verticalmente en cada Estado miembro para facilitar la competencia, estos tienen discrecionalidad a la hora de decidir exactamente cómo lo hacen.

Los últimos paquetes también obligan a crear agencias de regulación independientes del gobierno, pero también en esto los estados tienen mucha libertad en cómo hacerlo mientras exista alguna agencia separada.

Finalmente, tanto en telecomunicaciones como en electricidad no hay todavía un mercado europeo realmente integrado ni una autoridad fuerte a nivel europeo. Estos son los aspectos que hoy distinguen Europa de Estados Unidos, especialmente en telecomunicaciones, como se explica en Vogelsang (2014). En Estados Unidos un número limitado de operadores de telecomunicaciones (operando en internet, televisión por cable y telefonía móvil) están presentes en todo el país/continente, sin barreras de marca, espectro radioeléctrico o licencias. En este país también hay una menor preocupación por asegurar el acceso de las empresas entrantes a las infraestructuras locales de la empresa establecida, y se favorece más la competencia entre empresas de telecomunicaciones verticalmente integradas. En Europa mientras tanto, a pesar de que existen criterios convergentes para favorecer la competencia y la entrada de operadores en otros países, los mercados, las licencias y la gestión del espectro todavía giran alrededor de los Estados miembros. Se da la paradoja de que la liberalización, como suele pasar, ha dado lugar a una oleada de fusiones y adquisiciones, de manera que los operadores resultantes, no necesariamente los más eficientes por la intervención de condicionantes políticos en el mercado de control corporativo, hoy se encuentran en multitud de mercados, tanto en telecomunicaciones como en electricidad; pero estos pocos operadores, a pesar de que por su capacidad financiera y tecnológica podrían hacerlo, no compiten en un único mercado integrado, por lo menos en telecomunicaciones, sino en muchos mercados balcanizados (es decir, separados artificialmente por barreras políticas). En electricidad se dan niveles parecidos de integración regional a través de los estados, y en todo caso en Europa todavía es más alta la integración vertical de las empresas, con grandes países como Francia, donde la empresa dominante todavía está presente en todos los segmentos de la cadena de valor. La tabla 2 resume los parecidos y las diferencias entre Estados Unidos y Europa.

Tabla 2

\begin{tabular}{|l|c|c|c|c|}
\hline & \multicolumn{2}{|c|}{ Estados Unidos } & \multicolumn{2}{c|}{ Unión Europea } \\
\hline & Electricidad & Telecomunicaciones & Electricidad & Telecomunicaciones \\
\hline $\begin{array}{l}\text { Integración a través } \\
\text { de los estados }\end{array}$ & Media & Alta & Media & Baja \\
\hline Integración vertical & Baja & Alta & Media & Baja \\
\hline
\end{tabular}

Fuente: Elaboración propia. 
Un ejemplo de la combinación de problemas de eficiencia y redistributivos que se dan en el sector eléctrico lo encontramos en la reciente reforma de la electricidad en España, donde se ha intentado frenar el aumento del déficit que se había acumulado respecto a las empresas operadoras, repartiéndolo entre los consumidores, los contribuyentes y diferentes tipos de empresas. La solución resultante ha favorecido bastante a los inversores en las empresas tradicionales (especialmente Red Eléctrica de España -REE- y Endesa) frente a los inversores en las empresas de energías renovables (como Abengoa), como refleja el gráfico 1, donde se comparan las ganancias accionariales para los inversores en cada empresa desde que se inició la reforma eléctrica (inicios de 2012) hasta septiembre de 2014. Lógicamente, para tener una visión total de los efectos distributivos, habría que tener datos respecto a los efectos para diferentes tipos de contribuyentes y consumidores, pero es mucho más difícil conseguirlos. Es un ejemplo de la existencia de ganadores y perdedores en las políticas públicas, lo que explica la activación de estas empresas como grupos de interés.

\section{Gráfico 1}

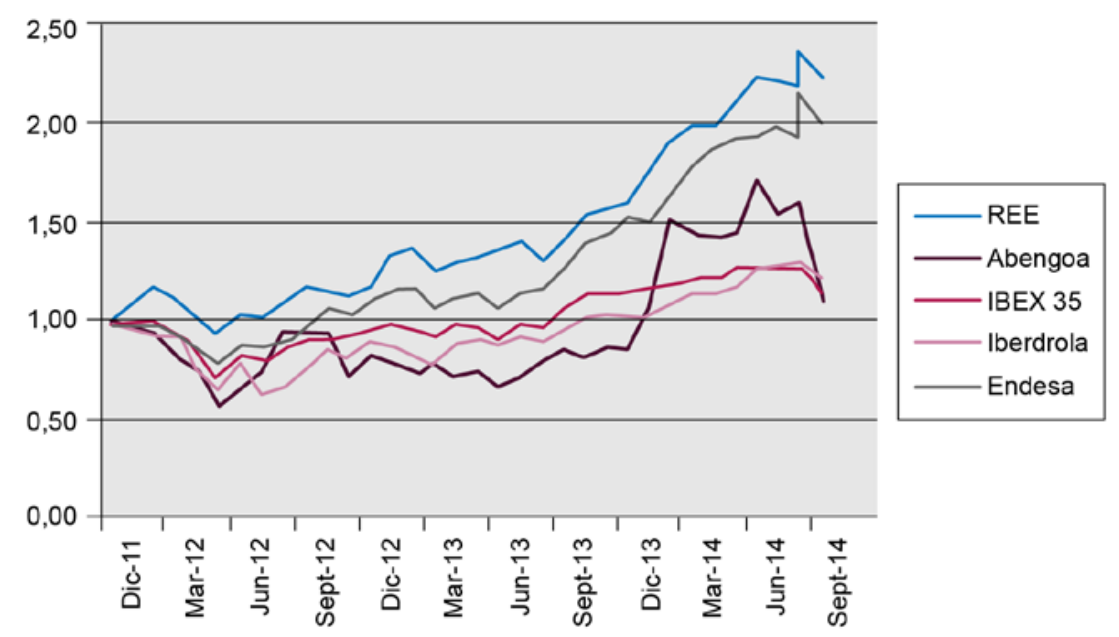

Fuente: Yahoo Finance y elaboración propia.

El caso español en electricidad ilustra las dificultades de resolver los dilemas (entre eficiencia productiva, eficiencia asignativa, efectos distributivos y solución a problemas medioambientales) que plantea el sector eléctrico en el marco tradicional del estado-nación. Dado el estado actual de desarrollo del proceso de integración europea, se plantea sin embargo la posibilidad de trasladar soberanía en cuanto a las políticas de este sector al nivel europeo. Así como en el siglo xIx en Estados Unidos se traspasó capacidad regulatoria de los municipios a los estados por razones políticas (y no tecnológicas), sería posible hoy y deseable traspasar más capacidad regulatoria de los Estados miembros de la Unión Europea a la Comisión Europea. En algunos casos esto está justificado por razones tecnológicas, por ejemplo en el caso de la necesaria coordinación del segmento de transporte en alta tensión de la electricidad y gestión de las actuales interconexiones entre redes. Pero incluso en aspectos como la producción de electricidad, la distribución o la prevención y eventual gestión de los déficits de tarifa, puede ser conveniente que se busque un árbitro lejos de las arenas políticas nacionales, donde los grupos de interés queden diluidos y la presión política sea menor. Quizá en este sentido paradójicamente puede ser beneficioso para la resolución de los dilemas que se presentan en el sector eléctrico que no esté demasiado desarrollado un demos europeo, aspecto que se presenta problemático desde otros puntos de vista. Un demos europeo consolidado implicaría la existencia de una arena política europea arraigada, con una opinión pública europea, unos partidos políticos europeos, unos lobbies operando a nivel europeo... La razón por la cual esta falta de desarrollo del demos europeo puede ser favorable en el sector eléctrico es que esto permitiría despolitizar las intervenciones públicas en sectores como el eléctrico, al menos durante el tiempo que tardara en desarrollarse el demos europeo, que 
previsiblemente puede ser largo dado el fuerte arraigo de los sentimientos nacionales todavía dentro de la Unión (si lo comparamos por ejemplo con los sentimientos locales en los estados de la confederación en Norteamérica en el siglo xvilI). La solución óptima a largo plazo sería que un demos europeo aceptara una regulación estable que permitiera unos niveles elevados de inversión y a la vez una solución a los problemas de cambio climático que tuviera en cuenta los intereses de las generaciones futuras.

\section{Conclusiones y comentarios finales}

A pesar de que la Unión Europea ha desempeñado ya un papel importante aumentando la competencia en las industrias de red, puede desempeñar -como hemos visto- un papel mucho más importante en el futuro avanzando hacia un auténtico mercado integrado donde existan redes realmente de alcance europeo, y donde los dilemas existentes se resuelvan a esta escala.

El plan Juncker de inversiones y la contribución a la solución de los problemas de cambio climático en la línea de lo que ha sido sugerido por el premio Nobel de Economía Jean Tirole (ver Tirole, 2012) son áreas donde la mayor integración puede dar mucho mejor resultado que la agregación de políticas nacionales.

Mientras tanto, sigue siendo una paradoja que lo único que cambia cuando atravesamos la línea divisoria entre un país y otro en la Unión Europea, por lo menos en el espacio Schengen y en la zona euro, es el sonido del teléfono móvil cuando identifica el cambio de compañía operadora.

\section{Referencias bibliográficas}

BRIGLAUER, W.; FRÜBING, S.; VOGELSANG, I. (2014). «The impact of alternative public policies on the deployment of new communications infrastructure -A surveym. Documento no publicado.

MAJÓ, J. (2015). «¿Costes fijos o variables?». El País. 7 de enero.

TIROLE, J. (2012). «Some Political Economy of Global Warming». Economics of Energy and Environmental Policy. Núm. 1(1), págs. 121-132.

TRILLAS, F. (2008). «Regulatory federalism in network industries». IEB Working Paper. 2008/8.

TRILLAS, F. (2010). «Electricity and telecoms reforms in the EU: Insights from the economics of federalism». Utilities Policy. Núm. 18, págs. 66-76.

VOGELSANG, I. (2014). "Will the US and EU telecommunications policies converge? A survey». CESifo Working Paper. Núm. 4843. 


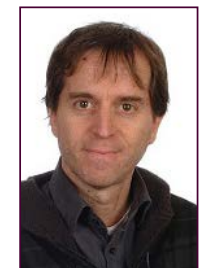

Francesc Trillas

francesc.trillas@uab.cat

Profesor agregado del Departamento de Economía Aplicada (UAB)

Profesor de la UAB e investigador del Centro Sector Público-Sector Privado del IESE y del Instituto de Economía de Barcelona. Obtuvo el doctorado en Economía en el Instituto Universitario Europeo de Florencia en diciembre de 2000, y fue investigador de la London Business School entre 1999 y 2002, así como investigador visitante en la Universidad de California-Berkeley en 2008. Ha escrito en varias publicaciones académicas internacionales sobre temas de regulación y economía política.

Los textos publicados en esta revista están -si no se indica lo contrario- bajo una licencia Reconocimiento-Sin obras derivadas 3.0 España de Creative Commons. Puede copiarlos, distribuirlos y comunicarlos públicamente siempre que cite su autor y la revista y la institución que los publica (autoría, nombre de la revista, institución editora); no haga con ellos obras derivadas. La licencia completa se puede consultar en http://creativecommons.org/licenses/by-nd/3.0/es/deed.es.

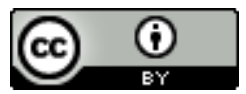

